\title{
Comunicación Horizontal: Identificación de la Demanda Social de Tecnología Agropecuaria en el Altiplano Peruano, Puno
}

\section{Horizontal Communication: Social Demand Identification of Agricultural Technology in the Peruvian Altiplano, Puno}

Fermín Francisco Chaiña-Chura ${ }^{1, \mathrm{a}}$ 0000-0003-0559-9748

Dante Salas-Avila ${ }^{1, b}$ 0000-0002-7033-2006

Marian Hermoza-Gutierrez ${ }^{2, \mathrm{c}}$ 0000-0003-1104-1695

Dante Salas-Mercado ${ }^{2, \mathrm{~d}}$ 0000-0003-0656-1979

Wenceslao Quispe-Borda ${ }^{1, \mathrm{e}} \underline{0000-0002-4842-3286}$

${ }^{1}$ Universidad Nacional del Altiplano, Puno, Perú.

${ }^{2}$ Programa de Doctorado en Ciencia, Tecnología y Medio Ambiente, Escuela de Posgrado, Universidad Nacional del Altiplano, Puno, Perú.

a fchaina@unap.edu.pe

bdsalas@unap.edu.pe

cmhermoza@unap.edu.pe

ddsalasm@unap.edu.pe

ewquispeb@unap.edu.pe
Recibido el: 26/07/2021

Aceptado el: 30/10/2021

Publicado el: 15/12/2021

\section{Resumen}

El objetivo de la investigación es identificar la demanda social de tecnología agropecuaria en el altiplano peruano como base para un proceso de comunicación horizontal: caso Centro Poblado de Santa Bárbara de Moro, en el departamento de Puno. Aplicando el método cuantitativo-no experimental, se encuestó a 80 jefes de familia para analizar la relación de 10 variables socioeconómicas con las demandas de tecnología agropecuaria. La actividad principal de los actores productivos es la crianza de ganado vacuno, cuyas demandas son predominantes con mayor incidencia en la implementación de cobertizos, sanidad animal, establos, máquinas ordeñadoras, entre otros; de igual forma, para el desarrollo de la pequeña agricultura, se evidenció la necesidad de adquisición de maquinaria agrícola y riego tecnificado. El estado civil, la actividad principal y la tenencia de ganado vacuno están altamente asociados a la demanda de tecnología al 99\% ( $p$-valor $<0.01$ ), mientras que el nivel de escolaridad tiene una significancia del 95\% (p-valor < 0.05). En conclusión, el estudio demuestra la importancia de la fase de identificación de la demanda de tecnología agropecuaria a partir de la comunicación horizontal, que contribuye al fortalecimiento de las actividades agroalimentarias.

Palabras clave: Actores productivos, agropecuario, comunicación horizontal, demanda tecnológica, identificación.

\begin{abstract}
The aim of this article is to identify the social demand for agricultural technology in the Peruvian highlands as the basis for a horizontal communication process, specifically in the Centro Poblado de Santa Bárbara de Moro (Puno). Applying the quantitative-nonexperimental method, eighty heads of households were surveyed out to analyze the relationship of ten socioeconomic variables with the agricultural technology demands. The main activity of the productive individuals is the cattle breeding whose demands are predominant with a greater incidence in the implementation of sheds, animal health, stables and milking machines, among others. Similarly, for the development of small agriculture, the need for the acquisition of agricultural machinery and technical irrigation was evident. The marital status, the main activity and the ownership of cattle are highly associated with the $99 \%$ demand for technology ( $p$-value $<0.01$ ), while the level of education has a significance of $95 \%$ (p-value $<0.05$ ). In conclusion, the study demonstrates the importance of the stage of identification of the demand for agricultural technology from the direct expression of the producers, as a basis for horizontal communication in agriculture, which contributes to the strengthening of agri-food activities.
\end{abstract}

Keywords: Productive actors, agriculture, horizontal communication, technological demand, identification. 


\section{Introducción}

La sociedad contemporánea es resultante del desarrollo global que ha producido profundos cambios sociales, científicos y tecnológicos; procesos que han tenido efectos en el medio rural (Arndt y Tarp, 2000). En este contexto, el direccionamiento de esfuerzos para la generación, transferencia y adopción (GTA) de soluciones tecnológicas ha tenido en las políticas públicas una diversidad de enfoques (Moreno-Valderrama et al., 2017) sin considerar una fase inicial importante de involucramiento del actor rural mediante la comunicación horizontal.

Las instituciones de investigación fueron diseñadas apuntando a la modernización de la agricultura vía transferencia de tecnología (Trigo y Elverdin, 2020). La generación de tecnología no es suficiente para obtener los impactos técnicos, económicos y sociales (Moctezuma-López et al., 2010). La mayor parte de los productores no adopta inmediatamente las tecnologías generadas en los centros de investigación, por ello es necesario tener conocimiento sobre la actitud del productor (López et al., 2019). Se muestra evidente el problema cuando se desarrolla el enfoque GTA de tecnología agropecuaria hacia los actores productivos, dejando de lado la fase inicial, es decir la identificación de la demanda de tecnología agropecuaria como base para la generación de innovaciones, transferencia y adopción (IGTA), no solo en las publicaciones, sino también en las políticas públicas.

En cuanto a los mecanismos de transferencia de tecnología, no existen instrumentos que permitan determinar la forma en que debe llevarse a cabo el proceso, basado en la identificación de necesidades tecnológicas (Paternina et al., 2019), que no dependen exclusivamente de la voluntad de los actores productivos sino de condiciones materiales propias de cada actor y de las características del contexto en el cual actúan (Montoya, 2008). Este vacío, donde el proceso GTA no considera la fase de identificación de tecnología agropecuaria limita la participación de los actores productivos.

Al desarrollar la fase inicial de identificación, el proceso resulta más sostenible porque viabiliza mayor articulación con los actores involucrados; en términos de Ceballos (2021), es importante la opinión de los sujetos de investigación para tener un enfoque más amplio y real del problema, lo cual contribuye a lograr el completo éxito en todo el proceso, ayudando a prevenir el gasto de tiempo y costo (Rhoades y Booth, 1982); además, aumenta la probabilidad de disminuir la pobreza en el sector rural (Chambers, 1985), otorgando la reducción completa del trabajo agropecuario, debido a una buena identificación de la demanda tecnológica (Diiro et al., 2021).

Antecedentes previos sobre la importancia de la comunicación horizontal como proceso participativo para el intercambio de saberes entre los técnicos institucionales y los actores productivos, indican que estos deben estar en sintonía al compartir el conocimiento científico y los saberes locales (Cadena-Iñiguez et al., 2018), concordante con Orozco et al. (2008), quienes consideran que el método de extensión participativa permite desarrollar capacidades que facilitan la adopción de tecnologías generadas localmente en la región Mazateca de Oaxaca México, consolidándose como un paradigma alternativo en extensión agrícola.

El objetivo de la investigación es identificar la demanda social de tecnología agropecuaria en el altiplano peruano como base para un proceso de comunicación horizontal en los actores sociales del Centro Poblado de Santa Bárbara de Moro, en el departamento de Puno.

\section{Marco Teórico}

\section{Teoría de la Demanda Social y Tecnología}

En un contexto económico, Bilas (1978) precisa que "la demanda se refiere a los bienes y servicios que los consumidores desean adquirir en un tiempo y precio determinado"; actualmente, se mantiene esta definición. Para Ipar y García (2021) la demanda social es la expresión de una carencia social, eso requiere siempre de cierta traducción a nivel social, en nombre de alguna colectividad, el barrio, la localidad, la comunidad, los pueblos originarios, entre otros. En un contexto social, se refiere a los requerimientos y urgencias de grupos humanos; en nuestro caso a las necesidades de los actores productivos hacia el sector institucional.

Relativo a la técnica y tecnología, PinedaHenao y Tello-Castrillón (2018) consideran que la técnica se refiere a los instrumentos que el hombre ha creado para la interacción con la naturaleza, a las habilidades requeridas 
para el uso de tales instrumentos con base a conocimientos y experiencia cotidianas, propias de su contexto cultural. Entonces, se considera que la técnica se circunscribe al problema de cómo podemos transformar la realidad. En tanto que, para Acevedo (2006), la dimensión técnica está asociada al significado más restringido de la tecnología, la dimensión organizativa y cultural, amplía el concepto de tecnología al considerar los valores que influyen en la actividad creativa de los diseñadores e inventores tecnológicos; concordante con Pineda-Henao y Tello-Castrillón (2018) al considerar que la tecnología se basa en el conocimiento científico contemporáneo, necesario para el diseño y producción de instrumentos.

La tecnología ha sido siempre un componente central de la vida humana y ha acompañado la evolución de las distintas culturas y civilizaciones (Cáceres, 2015). Esta práctica tecnológica está relacionada con la ciencia, entendida como un proceso sistemático; cuerpo de conocimientos ordenados en principios, leyes y teorías que explica el mundo; y una organización de científicos que usan métodos y técnicas para desarrollar nuevos conocimientos (Acevedo, 2006). El cambio tecnológico en el contexto de la historia cultural tiene un papel importante en la medida que se trate el cómo se origina, difunde, adopta e impacta la cotidianidad dicho cambio en la liberación de tiempo de trabajo (Garcia-Negrette, 2012).

Indudablemente, la importancia del conocimiento tecnológico en las sociedades contemporáneas y su creciente rol en diversas dinámicas productivas, constituye una temática ampliamente abordada en el contexto latinoamericano (Gárgano, 2018); además, en donde la agricultura es el motor del desarrollo rural, y el uso de tecnología está relacionado con el crecimiento de los países que se benefician de ella, pues resulta que la productividad es un factor determinante para satisfacer la demanda de alimento de la población (Osorio-García et al., 2012; Zand et al., 2019), por eso la tecnología disponible para los cultivos mejora su rendimiento y calidad si se utiliza en forma adecuada (Espinosa y Martínez, 2017).

\section{Desarrollo sostenible y teoría de la comunicación}

Dadas las condiciones que anteceden frente a la intensificación agraria, se hace hincapié en el desarrollo sostenible y el desarrollo rural (Larrubia, 2017). Sin desarrollo agropecuario no hay desarrollo rural y sin desarrollo rural no hay desarrollo sostenible (CEPAL, 2019; MINAGRI, 2016); en efecto, la sustentabilidad es la habilidad de lograr una prosperidad económica estable protegiendo los sistemas naturales, proveyendo una alta calidad de vida para las personas (Valarezo et al., 2020) y dinamizando el funcionamiento de las unidades familiares en el ámbito social, en el aspecto ambiental y en lo económico (Bedoya y Julca, 2021). En este mismo orden y dirección, la agricultura sustentable implica, entre otras cosas, conservación de los sistemas naturales a largo plazo, producción óptima con costos reducidos, adecuado nivel de ingreso, beneficio por unidad de producción y satisfacción de las necesidades alimentarias básicas (Valarezo et al., 2020).

La producción científica y tecnológica generada a partir de la investigación debería ser un recurso de flujo constante a disposición de la sociedad (Sánchez-Galán, 2020) y que se visibilice claramente a través de una serie de estrategias que promuevan la transferencia de nuevas innovaciones, conocimientos, tecnologías, prácticas y/o habilidades de un entorno a otro, es decir, de la investigación a la práctica. Sin embargo, el enfoque reconoce que una interacción débil entre los actores puede provocar bajos niveles de adopción tecnológica y afectar la calidad de los vínculos (Barboza y Sáenz, 2020).

Resulta oportuno comprender la naturaleza de los procesos del desarrollo agrario para elaborar estrategias viables de mejora (Cáceres, 2015). El conocimiento de la manera en que una empresa, finca o unidad productiva agrícola gestiona sus procesos y productos sirve de punto de partida para una proyección futura hacia un mejor desempeño y sostenibilidad en el proceso de gestión (López et al., 2019).

La comunicación desempeña un papel importante en los procesos de desarrollo rural, siendo necesaria la planificación de esta comunicación como un esfuerzo deliberado, sistemático y continuo que permita atender las prioridades de desarrollo de las comunidades, teniendo en cuenta sus formas predominantes de organización social y económica (Beltrán, 2007).

En el marco de una comunicación horizontal como proceso educativo no formal entre técnicos del sector institucional y actores productivos, Freire (1984) considera que no se trata de "llenar" al 
educando de "conocimiento" de orden técnico sino de proporcionar, a través de la relación dialógica, la organización de un pensamiento correcto en ambos, en una estructura horizontal y recíproca que no puede romperse. Así mismo, Barranquero y Sáez (2010) indican que la comunicación tradicional unilineal de la academia (fuente, mensaje, canal, receptor y efecto), va en contra de la comunicación alternativa y la comunicación para el cambio social, al considerar que, el carácter participativo en la construcción del conocimiento distribuye de modo más horizontal el saber en las comunidades. En la medida en que esta comunicación sea más efectiva, mayor será la posibilidad de convertir los conocimientos en una innovación práctica (Palmieri y Rivas, 2015).

\section{Metodología}

La metodología para la identificación de la demanda social de tecnología agropecuaria discurre por las siguientes fases: a) ámbito de estudio b) tipo de investigación y unidad de análisis, c) población y muestra, d) indicadores, técnicas e instrumentos y e) análisis de la información y método estadístico. Estos pasos se detallan en las secciones siguientes:

\section{Ámbito de estudio}

El ámbito de estudio forma parte del altiplano peruano, en el centro poblado de Moro (C.P. Moro) con coordenadas geográficas -70.067 long y -15.675 lat, ubicado en el distrito de Paucarcolla, a una altitud de 3,824 msnm. Estableciéndose en el piso altitudinal Suni, encontrándose en las proximidades del lago Titicaca. La actividad agrícola de la zona se caracteriza por la producción de cultivos de papa, quinua, entre otros; mientras que en la actividad pecuaria predomina la crianza de vacunos, ya que el área brinda condiciones favorables al estar cubierto de vegetación de regeneración natural (Hernández et al., 2020). En la figura 1 se observa que en el C.P. Moro se encuentran pastos de calidad agrológica media con riesgo de erosión, constituyendo el grupo de pastos aptos para pastoreo (MINAGRI, 2018); asimismo, existen pastos con una calidad agrológica media cuyas características exigen prácticas de manejo y conservación del suelo moderno y, además, presenta una limitación principal de erosión.
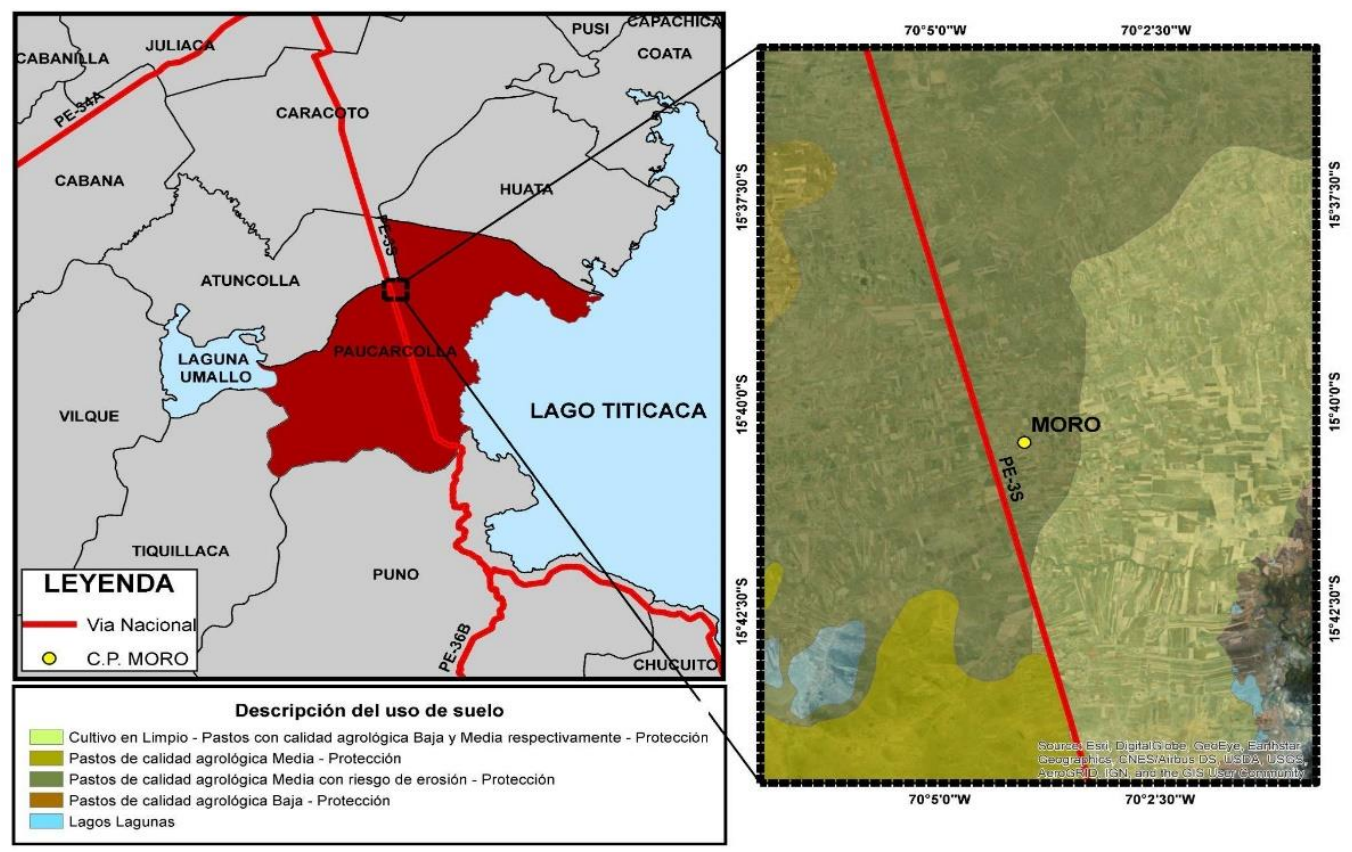

Figura 1. Localización geográfica del Centro Poblado Moro.

\section{Tipo de investigación y unidad de análisis}

El tipo de estudio está enmarcado dentro del método cuantitativo, no experimental; el diseño es correlacional y explicativo (Hernández et al.,
2014) debido a que se analiza la demanda social de tecnología agropecuaria utilizando procedimientos cuantitativos para verificar la relación existente entre las variables sociales, económicas y demanda social. La unidad de observación es el jefe de 
familia y la unidad de análisis es la demanda social de tecnología agropecuaria; concordante con Chacon et al. (2021) al precisar que la unidad de análisis es el objeto de estudio.

\section{Población y muestra}

La población del C.P. Moro según el Instituto de Estadística e Informática (INEI, 2017) está constituida por 105 familias dedicadas a la actividad agropecuaria y se determinó el tipo de muestra probabilística aplicando la siguiente fórmula:

$$
\mathrm{n}=\frac{\mathrm{z}^{2}(\mathrm{pq})}{\mathrm{e}^{2}+\frac{\left(\mathrm{z}^{2}(\mathrm{pq})\right)}{\mathrm{N}}}
$$

Donde: " $n$ " es el tamaño de la muestra, "N" es el tamaño de población, " $\mathrm{z}$ "es el nivel de confianza deseado de 1.96, "p" es la proporción de la población con la característica deseada (éxito) con 0.7 , "q" es la proporción de la población sin la característica deseada (fracaso) con un valor de $0.3 \mathrm{y}$ "e" es el nivel de error dispuesto a cometer con 0.05 . El valor de la muestra calculada a un $95 \%$ de confianza fue de 80 jefes de familia.

\section{Indicadores, técnicas e instrumentos}

La demanda social de tecnología agropecuaria se ha identificado aplicando la comunicación horizontal de ida y vuelta para la toma de datos mediante una encuesta semiestructurada, incidiendo en los aspectos sociales y económicos de las familias, así como el desarrollo de actividades principales; específicamente, se ha considerado las variables sociodemográficas referidas a indicadores personales, familiares y sociales; también se han considerado variables económico-productivas de tenencia de tierra, ganado, ingreso y la demanda de tecnología agropecuaria; en total se estiman cinco indicadores sociales y cinco económicos.

\section{Análisis de la información y método estadístico}

El análisis de la demanda social de tecnología agropecuaria se realizó haciendo uso del software de acceso libre $\mathrm{R}$ en dos niveles: a) análisis explicativo, para lo cual se clasificó, codificando y procesando la base de datos para estimar los promedios, distribución defrecuencias, porcentajes, entre otros estadísticos; b) la verificación de hipótesis, contrastando las propuestas, aplicando la prueba estadística de chi-cuadrado de Pearson que permite determinar si existe correlación entre las variables; las hipótesis estadísticas: Ho: Plantea que las variables no se encuentran asociadas. Ha: Plantea que las variables están asociadas. Regla de decisión: cuando el p-valor es menor a 0.05 indica que existe dependencia entre las variables.

\section{Resultados}

La interacción entre el equipo de investigación y los actores productivos se mantuvo enmarcada en el proceso de comunicación horizontal durante la recolección de la información requerida.

\section{Variables sociales}

Los resultados de las variables sociales se muestran en la tabla 1. El $65 \%$ de los jefes de familia son varones y el 35\% son mujeres. El estado civil presenta la predominancia de jefes de familia con la condición de conviviente y casados, conformando una familia nuclear; asimismo, se encuentran en menor proporción los viudos, divorciados y solteros. El nivel de escolaridad formal alcanzado por el jefe de familia, presenta predominancia en los niveles de primaria y secundaria en una proporción de $43.80 \%$ y $41.20 \%$ respectivamente. El $11.20 \%$ y $1.20 \%$ de jefes de familia cuentan con estudios superiores y tecnológicos; el $2.5 \%$ de los jefes de familia no tienen nivel educativo, por no asistir a un centro de educación formal o por olvido de lo aprendido en sus primeros años de escolaridad.

Tabla 1. Variables sociales de los jefes de familia del Centro Poblado de Moro.

\begin{tabular}{lccc}
\hline Variable & Frecuencia & Porcentaje & $\begin{array}{c}\text { Porcentaje } \\
\text { acumulado }\end{array}$ \\
\hline Género & 52 & 65.00 & 65.00 \\
Masculino & 28 & 35.00 & 100.00 \\
Femenino & & & \\
Estado civil & 8 & 10.00 & 10.00 \\
Soltero & 28 & 35.00 & 45.00 \\
Conviviente & 32 & 40.00 & 85.00 \\
Casado & 8 & 10.00 & 95.00 \\
Viudo & 4 & 5.00 & 100.00 \\
Divorciado & & & \\
\hline Grado de instrucción & & 2.50 & 2.50 \\
Sin estudios & 2 & 43.80 & 46.30 \\
Primaria & 35 & 41.20 & 87.50 \\
Secundaria & 33 & 1.20 & 88.50 \\
Técnico superior & 1 & 11.20 & 100.00 \\
Superior & 9 & & \\
\hline
\end{tabular}


Los resultados de las variables sociales continuas indican que el promedio del número de integrantes que conforman la unidad familiar es 4 personas; así mismo, los jefes de familia bordean los 47 años de edad en promedio (tabla 2).

Tabla 2. Variables económicas continuas y medidas de tendencia central.

\begin{tabular}{lcccccc}
\hline Estadísticos & Promedio & Mediana & SD & Varianza & Mínimo & Máximo \\
\hline $\begin{array}{l}\text { Número de } \\
\text { Integrantes }\end{array}$ & 4.23 & 4 & 1.88 & 3.52 & 1 & 12 \\
Edad & 46.78 & 46.5 & 13.68 & 187.14 & 18 & 85 \\
\hline
\end{tabular}

\section{Variables económicas}

En la tabla 3 se presentan las principales actividades productivas identificadas en Moro, entre las que se encuentra la ganadería, reflejando que un $63.70 \%$ de los jefes de familia encuestados se dedican a esa labor, seguida de las prácticas agropecuarias representadas por un $18.80 \%$. Una proporción reducida se dedica a la artesanía y otros quehaceres.

Tabla 3. Principales actividades productiva de los jefes de familia del Centro Poblado de Moro.

\begin{tabular}{lccc}
\hline Variable & Frecuencia & Porcentaje & $\begin{array}{c}\text { Porcentaje } \\
\text { acumulado }\end{array}$ \\
\hline Agricultura & 6 & 7.50 & 7.50 \\
Agropecuaria & 15 & 18.80 & 26.20 \\
Artesanía & 1 & 1.20 & 27.50 \\
Ganadería & 51 & 63.70 & 91.20 \\
Ganadería y producción de leche & 6 & 7.50 & 98.80 \\
Actividades en el hogar & 1 & 1.20 & 100.00 \\
\hline
\end{tabular}

La tabla 4 muestra los estadísticos del sondeo de variables económicas respecto a los datos cuantitativos que caracterizan a las actividades productivas. En general, los promedios reflejan que el ingreso económico de los actores encuestados es de 625.25 soles mensuales, el tamaño del predio familiar es de 3.11 hectáreas, el número de ganado vacuno supera las 12 unidades y el número de vacas productoras de leche bordea las 5 unidades en promedio.

Tabla 4. Variables económicas continuas y medidas de tendencia central.

\begin{tabular}{lcccccc}
\hline Estadísticos & Promedio & Mediana & SD & Varianza & Mínimo & Máximo \\
\hline $\begin{array}{l}\text { Ingreso Económico } \\
\text { (soles/mes) }\end{array}$ & 625.25 & 400 & 601.53 & 361832.85 & 20 & 3000 \\
$\begin{array}{l}\text { Tamaño de Predio (hectáreas) } \\
\text { Número de Ganado Vacuno }\end{array}$ & 3.11 & 1 & 5.1 & 25.97 & 0 & 30 \\
$\begin{array}{l}\text { (unidades) } \\
\begin{array}{l}\text { Número de vacas productoras } \\
\text { de leche (unidades) }\end{array}\end{array}$ & 12.13 & 10 & 9.72 & 94.54 & 0 & 55 \\
\hline
\end{tabular}

Se identificó que demanda de tecnología para las actividades productivas del Centro Poblado de Moro se centran en la implementación de mejoras para el desarrollo de la actividad pecuaria, siendo la principal demanda la adquisición de maquinaria agrícola $(33.80 \%)$ y otras sobresalientes como cobertizos, sanidad animal, establos y máquinas ordeñadoras para fortalecer la actividad pecuaria (tabla 5). 
Tabla 5. Demanda de tecnología agrícola, pecuaria y otros.

\begin{tabular}{lccc}
\hline Demanda Tecnológica & Frecuencia & Porcentaje & $\begin{array}{c}\text { Porcentaje } \\
\text { Acumulado }\end{array}$ \\
\hline Agua potable & 2 & 2.5 & 2.5 \\
Bebederos & 1 & 1.2 & 3.8 \\
Cerco eléctrico & 1 & 1.2 & 5 \\
Cobertizos & 10 & 12.5 & 17.5 \\
Comederos & 3 & 3.8 & 21.2 \\
Energía eléctrica & 2 & 2.5 & 23.8 \\
Especialista en lácteos & 1 & 1.2 & 25 \\
Establos & 5 & 6.2 & 31.2 \\
Ganadería & 3 & 3.8 & 35 \\
Inseminación artificial & 3 & 3.8 & 38.8 \\
Máquina de coser & 1 & 1.2 & 40 \\
Maquinaria agrícola & 27 & 33.8 & 73.8 \\
Maquinas ordeñadoras & 5 & 6.2 & 80 \\
Otra necesidad & 4 & 5 & 85 \\
Producción de forraje & 2 & 2.5 & 87.5 \\
Riego tecnificado & 3 & 3.8 & 91.2 \\
Sala de ordeño & 1 & 1.2 & 92.5 \\
Sanidad animal & 6 & 7.5 & 100 \\
\hline
\end{tabular}

\section{Nivel de asociación de las variables en estudio}

En la tabla 6 se presentan los resultados de la prueba chi-cuadrada de Pearson para verificar si hay asociación existente entre las variables socioeconómicas y la demanda de innovaciones tecnológicas.

Tabla 6. Relación entre variables socioeconómicas y demanda de tecnología agropecuaria.

\begin{tabular}{lccc}
\hline \multicolumn{1}{c}{ Chi-cuadrado de Pearson y $\left(\mathbf{d t}^{*}\right)$} & Valor & gl & p-valor \\
\hline Edad \& Necesidades de Tecnología $\left(\mathrm{dt}^{*}\right)$ & 698.24 & 663 & 0.166 \\
Sexo \& dt* & 21.701 & 17 & 0.197 \\
Estado Civil \& dt* & 106.39 & 68 & $\mathbf{0 . 0 0 2}$ \\
Nivel de Escolaridad \& dt* & 93.217 & 68 & $\mathbf{0 . 0 2 3}$ \\
Número de Integrantes \& dt* & 161.78 & 170 & 0.662 \\
Ingreso Económico \& dt* & 400.83 & 374 & 0.163 \\
Actividad Principal \& dt* & 143.14 & 85 & $\mathbf{8 . 2 0}$ e-05 \\
Tamaño de Predio \& dt* & 183.96 & 187 & 0.549 \\
Tenencia de Ganado Vacuno \& dt* & 566.78 & 459 & $\mathbf{4 . 2 9}$ e-04 \\
Vacas en Producción \& dt* & 255.77 & 221 & 0.054 \\
N $^{0}$ de Casos & 80 & & \\
\hline
\end{tabular}

Dt*. Demanda de tecnología agropecuaria.

Según los datos contenidos en la tabla 6 se determinó que, de las 10 variables socioeconómicas, 4 variables son representativas. El estado civil, la actividad principal y la tenencia de ganado vacuno están altamente asociados a la demanda de tecnología al 99\% ( $\mathrm{p}$-valor $<0.01$ ), mientras que el nivel de escolaridad tiene una significancia del $95 \%$ (p-valor $<0.05)$. 


\section{Discusión}

La dimensión de la inclusión social y económica en el proceso de difusión de tecnología se ha abordado sólo en raras ocasiones en la literatura, a pesar de su gran relevancia en las iniciativas de investigación y desarrollo. La noción de desarrollo inclusivo se está volviendo cada vez más popular en los círculos académicos y políticos, especialmente con la Agenda 21 y los Objetivos de Desarrollo Sostenible (United Nations Organization, 2017).

\section{Variables sociales}

El estado civil de los productores refleja su comportamiento como grupo social (Brumă et al., 2021), se observó que la mayoría gozan de una estabilidad civil, que los compromete más con su actividad productiva, pues tienen arraigo social mayor que los de otro estado civil (De la CruzLandero et al., 2015). La condición civil influye en la toma de decisiones sobre innovaciones tecnológicas como resultante del diálogo familiar, para emprender acciones futuras referentes al mejoramiento de su sistema de producción (Ardi y Brando, 2019; Bergamini et al., 2020; De la CruzLandero et al., 2015; Oliva et al., 2018).

La composición familiar está referida al número de integrantes. El grupo familiar de los productores está conformado por el padre, la madre, hijos y en algunos casos otros parientes concordantes con el estudio de De la Cruz-Landero et al. (2015); es una variable importante debido a que comprende la mano de obra que interviene en los procesos productivos en el interior del sistema de producción familiar, cuya resultante es el nivel de producción e ingreso económico que contribuye al mejoramiento del nivel de vida.

El nivel educativo formal alcanzado por el jefe de familia es otra variable importante debido a que, el mayor nivel educativo puede contribuir al requerimiento de mayor nivel tecnológico (Alarcón et al., 2018). El nivel de escolaridad está asociado a procesos de fortalecimiento de capacidades, al respecto Oliva et al. (2018) afirman que las personas con mayor nivel educativo fueron las que adoptaron las tecnologías; es decir, cuanto más conocimiento y mayor información tuvieron los productores, mayores fueron las posibilidades de adopción de las tecnologías (Montero Delgado et al., 2020).
Los productores no pueden innovar con la eficiencia y eficacia que el proceso exige; necesitan capacidad complementaria que no está en el grupo de productores, gran parte del conocimiento formal se encuentra en universidades e institutos de investigación (Bergamini et al., 2020), por lo que se requiere inversión del sector público en investigación y desarrollo para el fortalecimiento de capacidades productivas y gestión de su unidad de producción familiar.

\section{Variables económicas}

La actividad principal de los actores productivos del Centro Poblado de Moro se centra en la ganadería y la pequeña agricultura, con relieve en la crianza de ganado vacuno, la producción leche y derivados lácteos (tabla 4). Esto concuerda con estudios previos en el altiplano peruano (Chaiña e Inquilla, 2019; Cochi et al., 2014), constituyéndose la estructura y funcionamiento de los sistemas de producción familiar en ámbitos rurales; las variables económicas están vinculadas a las actividades productivas, en donde el medio de producción básico y fundamental es el recurso tierra, referidas a la existencia de pastos naturales, así como espacios amplios para pastos cultivados. Un indicador importante de las economías familiares es el tamaño de la propiedad de la tierra, que varía hasta un máximo de 30 hectáreas. Estas unidades familiares constituyen, en términos conceptuales, economías campesinas de subsistencia, debido a que producen para el consumo de la familia y para el mercado.

La crianza de ganado vacuno es importante debido a que genera ingresos económicos por la venta de leche o a través del procesamiento de lácteos como quesos de diferentes variedades, mantequilla y yogurt, que se comercializan en mercados locales, así como en las principales ciudades de la región y en mercados extrarregionales. Sin embargo, a pesar de su potencial, el sector no ha tenido el desempeño esperado debido a una serie de desafíos que persisten a través de los años, entre los cuales se encuentran la falta de habilidades empresariales, tecnológicas e innovadoras necesarias (Chipfupa y Wale, 2020; Sinyolo et al., 2017) y las estructuras de apoyo para el desarrollo financiero local (Montoya-Zumaeta et al., 2021). 


\section{Demanda social de tecnologías agropecuarias}

Las demandas tecnológicas que expresan los jefes de familia se han basado principalmente en cubrir las necesidades que podrían contribuir al desarrollo pecuario: cobertizos, sanidad animal, establos, máquinas ordeñadoras; coincidente con Paternina et al. (2019) y Martin (2021), al referir que el problema radica principalmente en la inadecuada gestión, dada la no implemetación de las buenas prácticas ganaderas - BPG, en términos de: instalaciones pecuarias y bienestar animal, alimentación y medio ambiente, sanidad animal y bioseguridad, entre otros.

Debido al constante requerimiento de alimento para el pastoreo y las características cultivables de la tierra, los productores demandan tecnologías relacionadas a la agricultura como segunda preferencia: maquinaria agrícola y riego tecnificado, que les permitan desarrollar actividades económicas sostenibles y de subsistencia. Junto a las demandas identificadas se expresa que estos productores necesitan de conocimientos tecnológicos para dar eficiencia a su sistema de producción en perspectiva del desarrollo sostenible, opiniones concordantes con los estudios de (Bedoya Justo \& Julca Otiniano, 2021; De La Cruz S. \& Dessein, 2021; Valarezo et al., 2020; Wordofa et al., 2021).

\section{Variables socioeconómicas asociadas a la demanda tecnológica}

290 La variable estado civil está asociada a la demanda tecnológica, ya que los núcleos familiares formados por convivientes y casados demandan tecnología agropecuaria que ayudaría a facilitar su trabajo y a no descuidar las obligaciones del hogar (Escalante, 2019; Ruzzante et al., 2021) como también, el nivel de escolaridad es una variable asociada a la demanda tecnológica, ya que los jefes de familia poseen educación básica regular, la que comprende primaria y secundaria (INEI, 2017), por lo que las instituciones del estado como la dirección regional de agricultura realizan programas de extensión agrícola y ganadera para actualizarlos e instruirlos en mejorar su producción agropecuaria (AGRORURAL, 2020), que tiene por objetivo el fortalecimiento de capacidades para el desarrollo de comunidades rurales. Existe poca población con nivel educativo técnico superior en la zona y se infiere que es debido a que estos migran buscando oportunidades laborales/profesionales.
La tenencia de ganado vacuno es otra variable que tiene relación con la demanda de tecnología agropecuaria, concordante con Oliva et al. (2018), quienes mencionan que la actividad principal de crianza de vacunos aumenta la probabilidad de adopción de tecnologías. En los últimos años, la tecnología se ha desarrollado sorprendentemente. El sector rural no ha estado ajeno a este proceso (Cáceres, 2015), el sectorinstitucional sevelimitado en sus tareas debido a la precaria vinculación con agricultores y asociaciones de estos (Samaniego, 2000; Wossen et al., 2019). En este contexto, los emprendimientos en algunas economías familiares constituyen iniciativas individuales que se originan dentro del entorno comunitario (Morales-Juárez y Méndez-García, 2021), por lo que es evidente la necesidad de tecnología para inyectar mayor eficiencia a la gestión agropecuaria.

\section{Nueva ruta IGTA, base de la comunicación horizontal}

La promoción de la etapa de identificación para reconocer la importancia de esta fase inicial, basada en la evidencia, entre otras soluciones, podría allanar el camino para mejorar el desempeño de los pequeños ganaderos y agricultores en contextos que comparten características económicas, sociales y culturales y ampliar los impactos de las intervenciones de desarrollo rural en el altiplano peruano.

La demanda social de tecnología identificada en este estudio requiere el emprendimiento de actividades de investigación tecnológica que siga esta nueva ruta, diferente de lo tradicional, de la investigación a la práctica; un flujo constante a disposición de la sociedad (Sánchez-Galán, 2020). El proceso de generación de tecnología en el predio del productor o en el ámbito de la comunidad es un factor importante en la comunicación y adopción del conocimiento; con participación de los miembros de las familias (Sangerman-Jarquín et al., 2012); además, para mejorar las condiciones de vida de los pequeños productores, es necesario promover prácticas que permitan a los ganaderos tener mayor producción con menor área cultivada (Ferguson y Griffith, 2004).

De acuerdo con los razonamientos de Cáceres (2015) y López et al. (2019), mencionan que en la implementación de esta estrategia, el sujeto dinámico sea el pequeño productor agropecuario, que su participación sea enmarcada en la 
comunicación horizontal desde la fase inicial de identificación de la demanda de tecnología, así como en todo el proceso de generación de tecnología y en la socialización, que en algunos casos sea el comunicador, el que transmite hacia los otros actores productivos, con el propósito de hacer más sensible este proceso de adopción de tecnología generada localmente, debido a que, en el mundo cultural andino, es admirable que un miembro de la comunidad se convierta en el expositor que comparta las experiencias de este proceso de educación no formal y promueva la deliberación entre técnicos y los actores productivos.

Las unidades familiares juegan un papel clave, a largo plazo, en el mantenimiento de la economía en zonas rurales debido a su conocimiento de la producción local, a su capacidad de adaptación $\mathrm{y}$ a que sus conocimientos se transmiten por generaciones (Zepeda et al., 2020). Los saberes se construyen y reconstruyen a través del tiempo, cambian generacionalmente de acuerdo a las propias necesidades de los actores productivos.

Cuando el enfoque para determinar las demandas del productor es de tipo GTA, el actor del medio rural lo percibe como una concepción impositiva vertical de origen estatal, como una orden de tipo conductista. Cuando se incluye la etapa de identificación (IGTA), el productor se asume como sujeto activo, se considera incluido, pues la comunicación es horizontal y dialógica de ida y vuelta, lo que posibilita mejorar y viabilizar el proceso completo. En algunos casos, el Estado debe participar directamente y a través de políticas públicas previamente deliberadas y consensuadas que den respuesta a las demandas de los actores productivos (Espinosa y Martínez, 2017).

\section{Conclusiones}

La identificación de la demanda de tecnología de los actores productivos en el altiplano de Puno es predominante en los criadores de ganado vacuno lechero, dada por las condiciones favorables en los aspectos sociales, económicos y ambientales para el desarrollo de la crianza de ganado vacuno, especialmente en reproducción, alimentación, sanidad y manejo. En tanto que, para la agricultura, demandan maquinaria agrícola, riego tecnificado y proceso de producción de cultivos.

El estado civil, la actividad principal y la tenencia de ganado vacuno y el nivel de escolaridad están asociados a la demanda social de tecnología, lo cual contribuye al desarrollo tecnológico de unidades familiares rurales de manera funcional, dinámica y horizontal, que integre la participación de los interesados en todo el proceso IGTA. Esta fase inicial del proceso constituye la base para el establecimiento de políticas de investigación y generación de tecnologías agropecuarias, en perspectiva del desarrollo sostenible.

El estudio demuestra la importancia de la fase de identificación de la demanda de tecnología agropecuaria a partir de la expresión directa de los productores, como base de una comunicación horizontal en el agro, contribuyendo al fortalecimiento de las actividades agroalimentarias y constituyendo una buena alternativa en los procesos de comunicación para el desarrollo en contextos rurales.

\section{Agradecimientos}

Los autores agradecen a la Universidad Nacional del Altiplano de Puno, al Vicerrectorado de Investigación y reconocen el apoyo financiero del Proyecto Concytec - Banco Mundial "Mejoramiento y Ampliación de los Servicios del Sistema Nacional de Ciencia, Tecnología e Innovación Tecnológica" 8682-PE, a través de su unidad ejecutora ProCiencia (contrato número 01-2018-FONDECYT/BM-Programas de Doctorados en Áreas Estratégicas y Generales).

\section{Referencias}

Acevedo, J. A. (2006). Modelos de Relaciones entre Ciencia y Tecnología: un Análisis Social e Histórico. Revista Eureka Sobre Enseñanza y Divulgación de Las Ciencias, 3(2), 198-219.

AGRORURAL. (2020). Noticias Categoría Puno. https://www.agrorural.gob.pe/sede/direccionzonal-puno

Alarcón, A. M., Chahin, C., Muñoz, S., Wolff, M. y Northland, R. (2018). Perfil de Personas con Infección por VIH/SIDA: Diferencial Étnico, Económico y Socio-Cultural en Chile. Revista Chilena de Infectología, 35(3), 276-282. $\underline{10.4067 / \mathrm{s} 0716-10182018000300276}$

Ardi, R. \& Brando, R. M. (2019). Household Consumer Behavior in Disposing WEEE (Waste Electrical and Electronic Equipment). 
Proceedingsofthe5thInternationalConference on Industrial and Business Engineering, 96100. $10.1145 / 3364335.3364338$

Arndt, C. \& Tarp, F. (2000). Agricultural Technology, Risk, and Gender: A CGE Analysis of Mozambique. World Development, 28(7), 1307-1326. 10.1016/ S0305-750X(00)00017-6

Barboza, L. M. y Sáenz, F. (2020). La Gestión del Conocimiento para la Innovación y el Desarrollo Rural: Yulök Revista de Innovación Académica, 4(1), 21-34. 10.47633/yulk. v4i1.199

Barranquero, A. y Sáez Baeza, C. (2010). Comunicación alternativa y comunicación para el cambio social democrático: sujetos y objetos invisibles en la enseñanza de las teorías de la comunicación. Congreso Internacional AE-IC Málaga 2010 "Comunicación y desarrollo en la era digital". 1-25. https://aeic.org/malaga2010/upload/ok/453.pdf

Bedoya, E. y Julca, A. (2021). Sustentabilidad de las Fincas de Palto (Persea Americana Mill.) En la Región Moquegua, Perú. RIVAR, 8(22), 36-50. 10.35588/rivar.v8i22.4770

Beltrán, L. R. (2007). Un adiós a Aristóteles: La comunicación "horizontal". Punto Cero. Universidad Católica Boliviana. 12(15), 7191. http://www.scielo.org.bo/pdf/rpc/v12n15/ v12n15a09.pdf

Bergamini, S., Pinheiro, M., Martinez-Sánchez \& Romeiro, M. do C. (2020). Trust and Knowledge Contribution to Agricultural Cluster Performance. International Journal of Productivity and Performance Management. 4(1), 1-21. 10.1108/IJPPM-10-2019-0495

Bilas, R. (1978). Teoría microeconómica (3ra edición). Alianza, 420-465

Brumă, I. S., Vasiliu, C. D., Rodino, S., Butu, M., Tanasă, L., Doboș, S., Butu, A., Coca, O., \& Stefan, G. (2021). The Behavior of Dairy Consumers in Short Food Supply Chains during COVID-19 Pandemic in Suceava Area, Romania. Sustainability, 13(6), 30723076. 10.3390/su13063072
Cáceres, D. M. (2015). Tecnología Agropecuaria y Agronegocios. La Lógica Subyacente del Modelo Tecnológico Dominante. Tecnología Agropecuaria y Agronegocios, 16(31), 15-16. https://ri.conicet.gov.ar/handle/11336/9038

Cadena-Iñiguez， P., Guevara-Hernández， F., Argüello-Aguilar, R. \& Rendón-Medel, R. (2018). Communication process, extension and adoption of the technologies. Revista Mexicana de Ciencias Agrícolas, 9(4), 851864. 10.29312/remexca.v9i4.1401

Ceballos Pérez, S. G. (2021). Impacto Territorial del Manejo Forestal Comunitario en la Sierra Norte de Puebla, 2003-2017. Cuadernos de Desarrollo Rural, 17(86), 2002-2017. 10.11144/javeriana.cdr17.itmf

CEPAL. (2019). Perspectivas de la Agricultura y del Desarrollo Rural en las Américas: una Mirada hacia América Latina y el Caribe 2019-2020. Comisión Económica Para América Latina y El Caribe. https:// www.cepal.org/es/publicaciones/451-411perspectivas-la-agricultura-desarrollo-ruralamericas-mirada-america-latina

Chacón Guevara, R. A., Flores Mamani, E., Valencia Blanco, D. S. y Quispe Cornejo, N. (2021). Conflictos Socioambientales en el Proyecto Gasoducto Sur Peruano Provincia de Canas-Cusco.Comuni@cción: Revistade Investigación En Comunicación y Desarrollo, 12(1), 15-24. 10.33595/2226-1478.12.1.469

Chaiña, F. y Inquilla, J. (2019). Necesidades de Tecnología Agropecuaria en el Centro Poblado de Accaso - Puno, Perú. Ciencia \& Desarrollo, 18(24), 27-37. $\underline{10.33326 / 26176033.2019 .24 .782}$

Chambers, R. (1985). Agricultural Research for Resource-Poor Farmers: The Farmer-Firstand-Last Model. Agricultural administration, 20(1), 1-30. 10.1016/0309-586X(85)90063-9

Chipfupa, U. \& Wale, E. (2020). Linking Earned Income, Psychological Capital and Social Grant Dependency: Empirical Evidence from Rural KwaZulu-Natal (South Africa) and Implications for Policy. Journal of Economic Structures, 9(1), 1-18. 10.1186/s40008-02000199-0 
Cochi, N., Prieto, G., Dangles, O., Rojas, A., Ayala, C., Condori, B. y Casazola, J. L. (2014). Metodología para Evaluar el Potencial Productivo y la Dinámica Socioecológica de la Ganadería en Bofedales Altoandinos. Ecología en Bolivia, 49(3), 120-131.

Damba, O. T., Kodwo Ansah, I. G., Donkoh, S. A., Alhassan, A., Mullins, G. R., Yussif, K., Taylor, M. S., Tetteh, B. K. \& AppiahTwumasi, M. (2020). Effects of Technology Dissemination Approaches on Agricultural Technology Uptake and Utilization in Northern Ghana. Technology in Society, 62, 101-104. 10.1016/j.techsoc.2020.101294

De la Cruz-Landero, E., Córdova-Ávalos, V., García-López, E., Bucio-Galindo, A. y Jaramillo-Villanueva, J. L. (2015). Manejo Agronómico y Caracterización Socioeconómica del Cacao en Comalcalco, Tabasco. Foresta Veracruzana, 17(1), 33-40.

De La Cruz S., M. \& Dessein, J. (2021). Beyond Institutional Bricolage: An 'Intertwining Approach' to Understanding the Transition Towards Agroecology in Peru. Ecological Economics, 18, 91-107. 10.1016/j. ecolecon.2021.107091

Diiro, G. M. y Fisher, M. (2021). No How Does Adoption of Labor Saving Agricultural Technologies Affect Intrahousehold Resource Allocations? The Case of PushPull Technology in Western Kenya. Food Policy, 14(1) 102-104. 10.1016/j. foodpol.2021.102114

Escalante, W. (2019). Factores que Influyen en la Producción de Quinua Orgánica en la Parcialidad de Salahuma Juntuma, Distrito de Huancané, Periodo 2016-2017. Repositorio Institucional. Universidad Nacional Del Altiplano, 12-43.

Espinosa, E. G. y Martínez, M. Á. (2017). El Crédito Agropecuario en México. Revista Mexicana de Ciencias Agrícolas, 8(1), 179181. 10.29312/remexca.v8i1.81

Ferguson, B. G. y Griffith, D. M. (2004). Tecnología Agrícola y Conservación Biológica en El Petén, Guatemala. Manejo Integrado de
Plagas y Agroecología (Costa Rica), 72(72), 72-85.

Freire, P. (1984). ¿Extensión o comunicación?: La Concientización en el Medio Rural. 13va ed. México: Siglo XXI, 110-119.

Garcia-Negrette, R. (2012). Historia de la Tecnología Agropecuaria y su Desarrollo para los frutales en el Valle del Cauca. Magazin Empresarial, 8(17), 59-70.

Gárgano, C. (2018). Ciencia, tecnología y mercado: Investigaciones en arroz en el INTA argentino. Journal of Technology Management and Innovation, 13(1), 75-83. 10.4067/S0718$\underline{27242018000100075}$

Hernández, R., Fernández, C. y Baptista, P. (2014). Metodología de la investigación. In McGrawHill/Interamericana Editores (Ed.), México DF (Sexta Edición), 12-34.

Hernández, O., Mancilla, O. R., Palomera, C., Olguín, J. L., Flores, H., Can, Á., Ortega, H. M. y Sánchez, E. I. (2020). Evaluación De La Calidad Del Agua Y De La Ribera En Dos Cuencas Tributarias Del Río Tuxcacuesco, Jalisco, México. Revista Internacional de Contaminación Ambiental, 36(3), 689-701. $\underline{10.20937 / \text { rica. } 53595}$

INEI. (2017). Directorio Nacional de Centros poblados. Censos Nacionales 2017: XII de Población, VII de Vivienda y III de Comunidades Indígenas. https://www. inei.gob.pe/media/MenuRecursivo/ publicaciones digitales/Est/Lib1541/index. $\underline{\mathrm{htm}}$

Ipar, M. C. y García, I. (2021). El Pueblo como Comunidad de Afectos. Demanda Social y Desborde de la Democracia Liberal en la Teoría Política Populista. Mediações Revista de Ciências Sociais, 26(1), 50-58. $\underline{10.5433 / 2176-6665.2021 v 26 n 1 p 50}$

Larrubia, R. (2017). La Política Agraria Común y sus Reformas: Reflexiones en Torno a la Reforma de 2014-2020. Cuadernos Geográficos, 56(1), 124-147. http:// revistaseug.ugr.es/index.php/cuadgeo/article/ view/4634/5312 
López, M. I., Estrada, J. A., Elidea, R. y Moreno, R. R. (2019).Aproximación Teórica y Aplicada al Modelo de Diversificación Integral de Cultivos para el Desarrollo Agrícola y Económico en el Cantón Quinidé, Provincia de Esmeraldas, República del Ecuador. Mikarimin: Revista Cientifica Multidisciplinaria, 6, 241-258.

López, G. A., Yañez, M. G., Velázquez, T. D. J., Tafoya, F. A. y López, C. A. (2019). Actitud del Productor Agrícola ante la Transferencia de Tecnología en la Región Centro de Sinaloa. Revista Mexicana de Ciencias Agrícolas, 10(6), 1457-1462. 10.29312/remexca. v10i6.1654

Martin, P. (2021). A Future-Focused View of the Regulation of Rural Technology. Agronomy, 11(6). 10.3390/agronomy 11061153

MINAGRI. (2016). Politica Nacional Agraria. http://minagri.gob.pe/portal/download/pdf/pagraria/politica-nacional-agraria.pdf

MINAGRI. (2018). Reglamento de Clasificación de Tierras por su Capacidad de Uso Mayor. Journal of Chemical Information and Modeling, 53(9), 1689-1699.

Moctezuma-López, G., Espinosa-García, J. A., Cuevas-Reyes, V., Jolalpa-Barrera, J. L., Romero-Santillán, F., Vélez-Izquierdo, A. y Bustos-Contreras, D. E. (2010). Innovación Tecnológica de la Cadena Agroalimentaria de Maíz para Mejorar su Competitividad: Estudio de Caso en el Estado de Hidalgo. Revista Mexicana de Ciencias Agrícolas, 1(1), 101-110.

Montero, J. A., Merino, F. J., Monte, E., Ávila, J. F. y Cepeda, J. M. (2020). Competencias Digitales Clave de los Profesionales Sanitarios. Educación Médica, 21(5), 338344. 10.1016/j.edumed.2019.02.010

Montoya-Zumaeta, J. G., Wunder, S. \& Tacconi, L. (2021). Incentive-Based Conservation in Peru: Assessing the State of Six Ongoing PES and REDD+ Initiatives. Land Use Policy, 108-116. 10.1016/j.landusepol.2021.105514

Montoya, O. (2008). El Estado de la Demanda Tecnológica del Sector Productivo en el Departamento de Risaralda: Una Provocación al Debate. Scientia Et Technica, 14(38), 277282. $10.22517 / 23447214.3773$

Morales-Juárez, H. \& del Carmen Méndez-García, E. M. (2021). Tensions in Governance from Below: Independent Water Systems in the Municipality of Huajuapan de León, Oaxaca, Mexico.TecnologíayCienciasDelAgua,12(3), 204-256. 10.24850/J-TYCA-2021-03-06

Moreno-Valderrama, M. N., Flórez-Martínez, D.-H., Uribe-Galvis, C. P. y Yepes-Vargas, L. A. (2017). Articulación de la Oferta y la Demanda en Ciencia, Tecnología e Innovación a través de Agendas Dinámicas Territoriales y Focos Prospectivos: Caso de Estudio Sector Agropecuario Colombiano. VIII Congreso Internacional En Gobierno, Administración y Políticas Públicas GIGAPP, 23-27.

Oliva, M., Santos, L., Roicer, C., Mestanza, V., Natividad, C. \& L, M. J. (2018). Evaluation of Border Irrigation in the Southern Uruguayan Soils Use of the WinSRFR Model: Preliminay Results. Agrociencia Uruguay, 22(2), 1-9. $\underline{10.31285 / \text { agro.22.2.9 }}$

Osorio-García, N., López-Sánchez, H., Gil-Muñoz, A., Ramírez-Valverde, B., Gutiérrez-Rangel, N., Crespo-Pichardo, G. y Montero-Pineda, Á. (2012). Utilización, Oferta Y Demanda De Tecnología Para Producción De Maíz En El Valle De Puebla, México. Agricultura, Sociedad y Desarrollo, 9(1), 55-69.

Orozco, S., Jiménez Sánchez, L., Estrella Chulím, N., Ramírez Valverde, B., Peña Olvera, B.V., Ramos Sánchez, A. y Morales Guerra, M. (2008). Escuelas de campo y adopción de ecotecnia agrícola. Ecosistemas. 17(2), 94102. http://revistaecosistemas.net/index.php/ ecosistemas/article/view/475

Palmieri, V. y Rivas, L. (2015). Gestión de información para la innovación tecnológica agropecuaria. Instituto Interamericano de Cooperación para la Agricultura, 3, 17-26.

Paternina, H., Linares, J. y Hernández, K. (2019). Transferencia de Tecnología y Conocimiento en el Sector Exportador de Bovinos. Ipsa Scientia,4(1), 10-20.10.25214/27114406.936

Pineda-Henao, E. F. y Tello-Castrillón, C. (2018). 
¿Ciencia, técnica y arte?: Análisis Crítico Sobre Algunas Posturas del Problema del Estatus Epistemológico de la Administración. Revista Logos, Ciencia \& Tecnología, 10(4), 112-130. 10.22335/rlct.v10i4.605

Rhoades, R. E. \& Booth, R. H. (1982). Farmerback-to-Farmer: A Model for Generating Acceptable agricultural technology. Agricultural Administration, 11(2), 127-137. $\underline{10.1016 / 0309-586 X(82) 90056-5}$

Ruzzante, S., Labarta, R., \& Bilton, A. (2021). Adoption of Agricultural Technology in the Developing World: A Meta-Analysis of the EmpiricalLiterature. World Development, 146, 105-599. 10.1016/j.worlddev.2021.105599

Samaniego Gaxiola, J. A. (2000). Limitantes para el Desarrollo y Transferencia de Tecnología Agrícola en la Región Lagunera. Revista Mexicana de Agronegocios, 4(6), 1-9.

Sánchez-Galán, E. (2020). La Investigación Universitaria: El Caso de la Facultad de Ciencias Agropecuarias de la Universidad de Panamá: 2007-2018. Revista Anual Acción y Reflexión Educativa, 45, 1-27.

Sangerman-Jarquín, D. M., Rangel, E. E., Villaseñor Mir, H. E., Navarro, A., Larqué, B. S., de la O Olán, M. \& Torres, R. (2012). Technology Transfer to Wheat Producers in Nanacamilpa, Tlaxcala. Revista Mexicana de Ciencias Agrícolas, 3(8), 1591-1604.

Sinyolo, S., Mudhara, M. \& Wale, E. (2017). The Impact of Social Grant Dependency on Smallholder Maize Producers Market Participation in South Africa: Application of the Double-Hurdle model Research methodology. South African Journal of Economic and Management Sciences, 20(1), $1-10$.
Trigo, E. J. y Elverdin, P. (2020). Los Sistemas de Investigación y Transferencia de Tecnología Agropecuaria de América Latina y el Caribe en el marco de los nuevos Escenarios de Ciencia y Tecnología. Revista Compromiso Social, 3, 116-127.

Valarezo, C. O., Julca-Otiniano, A. y Rodríguez, A. (2020). Evaluación de la Sustentabilidad de Fincas Productoras de Limón en Portoviejo, Ecuador. Revista RIVAR, 7(20), 108-120. $\underline{10.35588 / \text { rivar.v7i20.4485 }}$

Wordofa, M. G., Hassen, J. Y., Endris, G. S., Aweke, C. S., Moges, D. K. \& Rorisa, D. T. (2021). Adoption of improved agricultural technology and its impact on household income: a propensity score matching estimation in eastern Ethiopia. Agriculture and Food Security, 10(1), 1-12. 10.1186/ $\underline{\mathrm{s} 40066-020-00278-2}$

Wossen, T., Alene, A., Abdoulaye, T., Feleke, S. \& Manyong, V. (2019). Agricultural Technology Adoption and Household Welfare: Measurement and Evidence. Food Policy, 87, 10-17. 10.1016/j.foodpol.2019.101742

Zand, P., Mirzaie, H., Mehrabi, H. \& Nabieian, S. (2019). Analysis of Economic and Social Impact of Investment Development Policy in Agricultural Sector. Journal of Agricultural Science and Technology, 21(7), 1737-1751.

Zepeda, J. A. Z., Valverde, B. R., López, L. L. V. y Elizalde, S. P. (2020). La Pequeña Empresa Agrícola Familiar, la Producción de Maíz y la Línea de Bienestar en Puebla, México. Regiones y Desarrollo Sustentable, 20(39), $10-17$. 